\title{
Palatability and Preference of Gummi Formulations with Various Pharmaceutical Characteristics
}

\author{
Fumiaki Nakagaki, ${ }^{a, b}$ Shinya Uchida, ${ }^{* a}$ Shimako Tanaka, ${ }^{a}$ and Noriyuki Namiki ${ }^{a}$ \\ ${ }^{a}$ Department of Pharmacy Practice and Science, School of Pharmaceutical Sciences, University of Shizuoka; 52-1 \\ Yada, Suruga-ku, Shizuoka 422-8526, Japan: and ${ }^{b}$ Biologics CMC Research and Technology, Pharmaceutical \\ Research Lab., Kissei Pharmaceutical Co., Ltd.; 4365-1 Kashiwabara, Hotaka, Azumino, Nagano 399-8304, Japan. \\ Received December 17, 2017; accepted January 23, 2018
}

This study aimed to elucidate the appropriate physical characteristics that are clinically acceptable for gummi formulations. We prepared 11 placebo gummi formulations containing different amounts of gelatin and water and evaluated their penetration and restitution using a penetrometer and rheometer, respectively.

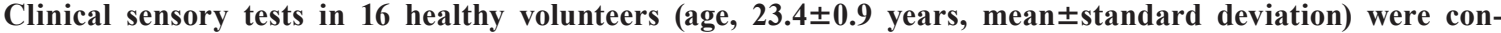
ducted on the placebo gummi formulations using the visual analog scale (VAS) score to evaluate elasticity, hardness, and overall palatability, with a 5-point rating scale of preference. The penetration increased with decreasing amounts of gelatin or increasing amounts of water in the gummi formulations. Similarly, the VAS score of elasticity and hardness from the clinical sensory tests increased with increasing amounts of gelatin but decreased with increasing amounts of water. The relationship between the penetration and VAS scores of elasticity and hardness revealed good linear correlations. This suggests that the penetration was well reflected by the hardness results of the clinical VAS scores. The overall palatability evaluated using the VAS score increased until the penetration was $10 \mathrm{~mm}$ and then plateaued at $>10 \mathrm{~mm}$ penetration. The 5-point rating score for preference revealed that $>\mathbf{5 0} \%$ of volunteers "prefer" the gummi formulations with penetration values of 9.8 to $13.5 \mathrm{~mm}$. These results suggest that gummi formulations likely have an appropriate window of hardness. Furthermore, appropriate gummi formulations with clinically preferred physical characteristics could be prepared by adjusting the amount of gelatin and water and measuring their penetration.

Key words gummi formulation; palatability; preference; clinical trial; physical characteristics

In pharmacotherapy, the medication adherence of patients is critical to ensuring the expected therapeutic effects are obtained and, therefore, should be improved. Various efforts have been made to improve medication adherence, ${ }^{1-3)}$ because it is a complex, multi-determined behavior that is often influenced by the cost of treatment, drug regimen complexity, and patient-related factors. Optimized dosage forms for each patient, such as orally disintegrating tablets, ${ }^{4,5)}$ oral jelly formulations, ${ }^{6)}$ chewable tablets, ${ }^{7,8)}$ and gummi formulations, ${ }^{9-11)}$ have important roles in medication adherence.

Gummi drugs are dried jelly drugs prepared by adding gelatin as a gelling agent to syrup, consisting of carbohydrates such as sugar and starch syrup that have been boiled down. Subsequently, the desired drugs are added to the syrup mixture, which is then cooled and allowed to solidified. ${ }^{11)}$ Gummi formulations are expected to improve adherence by enabling patients to swallow medications easily often without water. A gummi drug is a formulation that is to be chewed, similar to chewable tablets. The draft guidance for the U.S. Food and Drug Administration (FDA) chewable development stipulates that chewable tablets should be easy to chew, palatable (taste masked or have an acceptable taste), of an appropriate size and shape, readily disintegrate to minimize aspiration and facilitate dissolution. ${ }^{12)}$ These recommended properties should also be considered critical for gummi formulations. Gummi formulations need to be chewed, and the chewing force and movements may be strongly influenced by the texture of the formulation. ${ }^{13)}$ We successfully prepared gummi drugs of aripiprazole for use as hospital formulations; however, we do not know if they possessed the required clinically appropriate physical characteristics for use in clinical settings. ${ }^{11)}$

Therefore, we aimed to elucidate the appropriate physical characteristics that are acceptable for clinically administered gummi formulations. In this study, we prepared 11 placebo gummi formulations with different physical characteristics, and evaluated their elasticity, hardness, overall palatability, preference, chewing cycles, and the ease of swallowing using clinical sensory tests.

\section{Experimental}

Materials Gelatin (AP-50) and hydrogenated maltose starch syrup (Amalty syrup) were purchased from Nippi, Inc., (Tokyo, Japan) and Mitsubishi Shoji Foodtech Co., Ltd. (Tokyo, Japan), respectively. D-Sorbitol solution (75\%), anhydrous citric acid, and the other chemicals were of Japanese Pharmacopoeia (JP) grade.

Gummi Formulation Preparation Method We prepared 11 gummi formulations with different material attributes by adjusting the gelatin and water contents (Table 1). The procedure used to prepare the gummi formulations was a modified version of a previously described method ${ }^{11)}$ (Fig. 1). Amalty syrup and D-sorbitol solution were weighed, mixed, and then heated $\left(<135^{\circ} \mathrm{C}\right)$, to evaporate part of the water. The water contents of the gummi formulations were adjusted to five levels in this step by varying the amount of water evaporated. Then, an Amalty syrup/sorbitol mixture was prepared. Separately, water was added to make the gelatin swell, and then it was subsequently dissolved by heating to $60^{\circ} \mathrm{C}$.

The gelatin contents of the gummi formulations were adjusted to three levels in this step by adjusting the amount of 
gelatin solution added to the Amalty syrup/sorbitol mixture (L: low gelatin, $\mathrm{M}$ : moderate gelatin, $\mathrm{H}$ : high gelatin) after anhydrous citric acid solution was added. Then, the mixture was kept at $70^{\circ} \mathrm{C}$, with stirring and mixing. Finally, $3.5 \mathrm{~g}$ of this mixture was dispensed into a plastic plate shaped like a round pocket using a syringe, and cooled at room temperature $\left(15-25^{\circ} \mathrm{C}\right)$. For penetration measurement, the mixture was dispensed into a cylindrical beaker (inner diameter: $51.1 \mathrm{~mm}$ ) until the height and volume reached $37.5 \mathrm{~mm}$ and $80 \mathrm{~mL}$, respectively, using a syringe, and then it was cooled at room temperature $\left(15-25^{\circ} \mathrm{C}\right)$.

Measurements of Physical Properties of the Gummi Formulations We measured the penetration and restitution as physical properties of the gummi formulations. The penetration of the gummi formulation was measured using a penetrometer (Ikemoto Scientific Technology Co., Ltd., Tokyo, Japan). The needle tip of the instrument was placed in contact with the surface of the gummi formulation, and then a $50 \mathrm{-g}$ needle holder was used to penetrate the gummi drug with the needle for $5 \mathrm{~s}$. The penetrated distance $(\mathrm{mm})$ was defined as the penetration value.

The restitution of the gummi formulation was measured using a rheometer (Sun Scientific Co., Ltd., Tokyo, Japan). The tooth-shaped tip of the instrument touched the surface of the gummi formulation, and then the tip was allowed to push the gummi to a 7-mm distance. The peak force $(\mathrm{g})$ during the measurement was defined as the restitution value.

Measurement of Disintegration Time A disintegration test was conducted in accordance with the JP disintegration test method $(n=3)$ using a JP disintegration tester (Toyama Sangyo Co., Ltd., Osaka, Japan). The study was conducted with water as the disintegration medium.

Water Activities $\left(\boldsymbol{A}_{\mathrm{w}}\right)$ The $A_{\mathrm{w}}$ value was measured $(n=3)$ using a water-activated measurement device (ROTRONIC AG., Zurich, Switzerland). After confirming that the $A_{\mathrm{w}}$ value was $<0.200$ by placing silica gel in the sample chamber, the test sample was set in the sample chamber, and the device was closed. The reading was recorded after the $A_{\mathrm{w}}$ value was stable.

Clinical Sensory Test We conducted two trials in the clinical sensory test. A study of the placebo gummi formulations was conducted with 16 healthy volunteers (seven females and nine males; mean age \pm standard deviation (S.D.) $=23.4 \pm 0.9$ years) who participated in the study after providing written informed consent. The study protocol was approved by the Ethics Committee of the University of Shizuoka, Japan. The clinical sensory tests were conducted in a randomized crossover, single-blind trial, consisting of one stage that tested the texture of gummi formulations and the other that determined how many times the gummi needed to be chewed before it was swallowed, and how easy it was to swallow.

In the first trial, we conducted human clinical tests to evaluated elasticity, hardness, overall palatability of gummi formulations using the $100 \mathrm{~mm}$ visual analog scale (VAS) by placing a mark along a $100-\mathrm{mm}$ line, and to assess the preference using 5-point rating scale. The schedule of this trial is shown in Fig. 2. First, volunteers were asked to score the elasticity (0: not elastic, 100: very elastic) by picking a gummi formulation up. Then, they placed the gummi formulation in their oral cavities, and chewed for $20 \mathrm{~s}$. They evaluated hardness ( 0 : not hard at all, 100: very hard) and overall palatability (0: very bad, 100: very good) during chewing a gummi. All volunteers spat out the chewed gummi and rinsed their mouths out with water immediately. Then, all volunteers were asked to score their preference using the following 5-point rating scale: (1) I do not like it; (2) I do not like it very much; (3) I am just all right; (4) I like it; (5) I like it very much. "Prefer" was defined as options (4) and (5). The different gummi formulations were tested at 5-min intervals.

In the second trial, we conducted human clinical tests of the chewing cycles and the ease of swallowing the gummi formu-

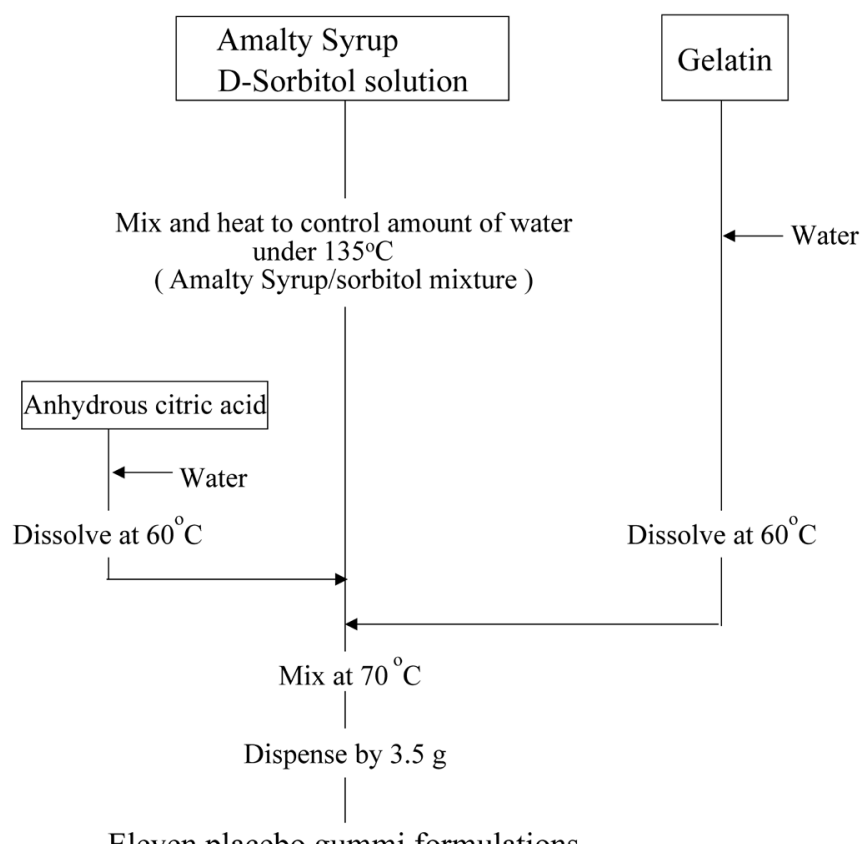

Eleven placebo gummi formulations containing different amounts of gelatin and water (L2-L4, M1-M5, H2-H4)

Fig. 1. Procedure for Preparation of Gummi Formulations

Amalty syrup: hydrogenated maltose starch syrup.

Table 1. Components of Gummi Formulations

\begin{tabular}{lrrrrrrrrrrrr}
\hline \hline \multicolumn{1}{c}{ Component (\%) } & L2 & L3 & L4 & M1 & M2 & M3 & M4 & M5 & H2 & H3 & H4 \\
\hline Gelatin & 3.4 & 3.4 & 3.4 & 6.9 & 6.9 & 6.9 & 6.9 & 6.9 & 13.7 & 13.7 & 13.7 \\
Hydrogenated maltose & 48.9 & 45.0 & 41.0 & 50.7 & 46.7 & 42.8 & 38.8 & 34.9 & 42.4 & 38.4 & 34.5 \\
$\quad$ starch syrup & & & & & & & & & & \\
D-Sorbitol solution & 27.9 & 25.6 & 23.4 & 28.9 & 26.6 & 24.4 & 22.1 & 19.9 & 24.1 & 21.9 & 19.6 \\
Anhydrous citric acid & 0.7 & 0.7 & 0.7 & 0.7 & 0.7 & 0.7 & 0.7 & 0.7 & 0.7 & 0.7 & 0.7 \\
Water & 19.1 & 25.3 & 31.5 & 12.9 & 19.1 & 25.3 & 31.5 & 37.7 & 19.1 & 25.3 & 31.5 \\
\hline Total & 100 & 100 & 100 & 100 & 100 & 100 & 100 & 100 & 100 & 100 & 100 \\
\hline
\end{tabular}


First trial

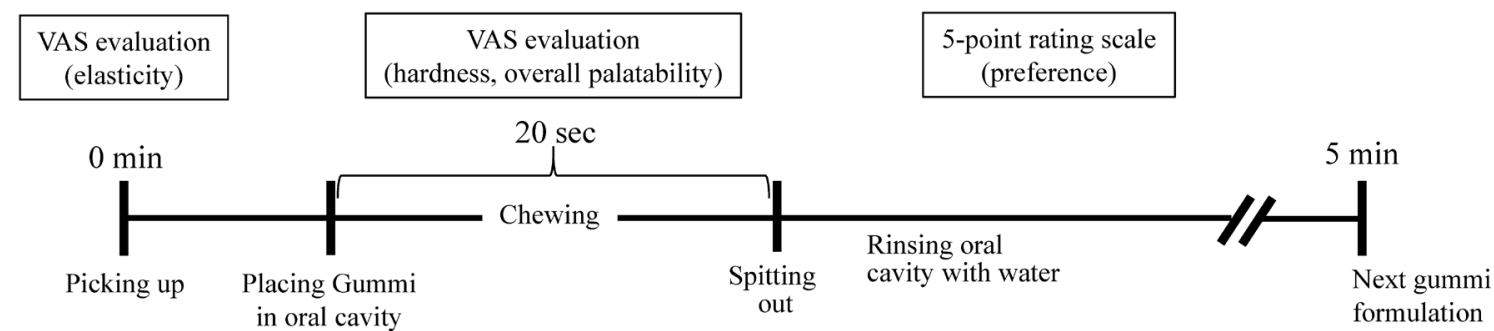

\section{$\underline{\text { Second trial }}$}

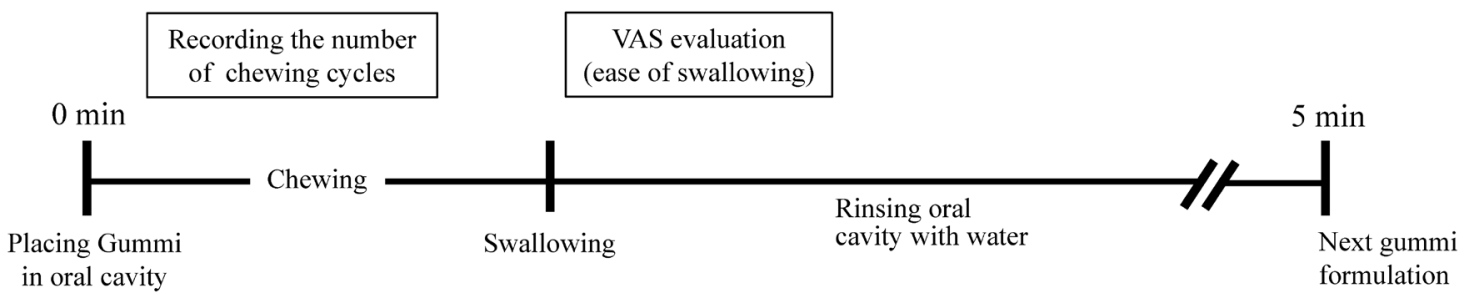

Fig. 2. Time Schedule of Human Sensory Tests

Table 2. Penetrated Distance, Restitution, Disintegration Time, and Water Activity $\left(A_{\mathrm{w}}\right)$ of Gummi Formulations

\begin{tabular}{ccccc}
\hline \hline Formulation & Penetrated distance $(\mathrm{mm})$ & Restitution $(\mathrm{g})$ & Disintegration time $(\mathrm{min})$ & Water activity \\
\hline L2 & $21.5 \pm 1.7$ & $124 \pm 22$ & $15.1 \pm 0.5$ & $0.675 \pm 0.005$ \\
L3 & $25.3 \pm 0.5$ & $48 \pm 13$ & $15.3 \pm 0.7$ & $0.760 \pm 0.008$ \\
L4 & $26.9 \pm 1.4$ & $39 \pm 3$ & $16.4 \pm 0.1$ & $0.819 \pm 0.012$ \\
M1 & $8.7 \pm 0.4$ & $275 \pm 57$ & $20.8 \pm 1.1$ & $0.582 \pm 0.002$ \\
M2 & $9.8 \pm 1.1$ & $300 \pm 56$ & $17.3 \pm 0.2$ & $0.674 \pm 0.004$ \\
M3 & $11.2 \pm 0.6$ & $176 \pm 9$ & $19.5 \pm 0.2$ & $0.772 \pm 0.009$ \\
M4 & $13.5 \pm 0.4$ & $132 \pm 9$ & $20.4 \pm 0.3$ & $0.829 \pm 0.010$ \\
M5 & $14.7 \pm 1.3$ & $101 \pm 20$ & $23.3 \pm 0.1$ & $0.881 \pm 0.010$ \\
H2 & $5.4 \pm 0.3$ & $286 \pm 34$ & $32.0 \pm 1.6$ & $0.737 \pm 0.004$ \\
H3 & $6.0 \pm 0.0$ & $279 \pm 4$ & $32.3 \pm 1.2$ & $0.799 \pm 0.008$ \\
H4 & $6.2 \pm 0.9$ & $229 \pm 9$ & $36.0 \pm 0.7$ & $0.855 \pm 0.011$
\end{tabular}

Data are means \pm S.D. of three subjects.

lations. The schedule of this trial is shown in Fig. 2. In this trial, all volunteers were asked to record the number of cycles needed to adequately chew the gummi formulations before swallowing. In addition, they were asked to evaluate the ease of swallowing (0: difficult to swallow, 100: easy to swallow) using the $100 \mathrm{~mm}$ VAS after a gummi had been chewed in their oral cavity. After the clinical VAS evaluation, all volunteers rinsed their mouth with water immediately. The different gummi formulations were tested at 5-min intervals.

Statistical Analysis The data are presented as the mean \pm S.D. except for the 5-point scale results, which are presented as the median and range. The statistical analysis was performed using the GraphPad Prism v. 5.02 software (GraphPad Software, San Diego, CA, U.S.A.).

\section{Results}

Material Attributes of Placebo Gummi Formulations Table 2 shows the penetration, restitution, disintegration time, and $A_{\mathrm{w}}$ of each gummi formulation. The penetration of the gummi formulations ranged from 5.4 to $26.9 \mathrm{~mm}$, and in- creased with increasing amounts of water. The restitution of the formulations ranged from 39 to $300 \mathrm{~g}$. A significant correlation $\left(R^{2}=0.811, p<0.001\right)$ was observed between the penetration and restitution (Fig. 3). The disintegration times of the gummi formulations ranged from 15 to $36 \mathrm{~min}$ and increased with increasing amounts of gelatin. The $A_{\mathrm{w}}$ values of the formulations ranged from 0.582 to 0.881 .

Measurement of Elasticity, Hardness, Overall Palatability and Preference in Healthy Volunteers Using VAS and 5-Point Rating Scale (First Trial) Figure 4 shows the VAS scores of the elasticity, hardness, and overall palatability of the gummi formulations. The VAS score of elasticity increased with increasing amounts of gelatin. Similarly, the VAS score of hardness increased with an increasing amount of gelatin but decreased with increasing amounts of water. The highest and lowest VAS scores of overall palatability were 74.0 and 25.3 for the M3 and H2 formulations, respectively. For the preference analysis, the M2, M3, and M4 formulations exhibited the highest median scores (4.0) while $>50 \%$ of volunteers responded "prefer," and the highest proportion (81\%) 
selected the M3 formulation (Table 3).

Measurement of Chewing Cycles and the VAS Scores of Ease of Swallowing (Second Trial) Table 3 shows the chewing cycles and VAS scores of ease of swallowing the gummi formulations. The chewing cycles increased with increasing amounts of gelatin. The lowest and highest mean chewing cycles were 18.9 and 51.8 for the L3 and H3 formulations, respectively. The VAS scores of ease of swallowing decreased with increasing amounts of gelatin. Although the volunteers swallowed the formulations after sufficient chewing, the higher gelatin content formulations (H2, H3, and H4) showed extremely low VAS scores of ease of swallowing of 47.8, 39.3, and 39.4, respectively.

Relationship between the Penetration and Clinical Sensory Tests Figure 5 shows the relationships between penetration and elasticity, hardness, and overall palatability. Good linear correlations were observed between penetration and VAS scores of elasticity and hardness $\left(R^{2}=0.864\right.$ and 0.924 , respectively; $p<0.001$ each). These results suggest that penetration was well reflected by the clinical VAS scores of the formulations in the human sensory test. However, no linear relationship was observed between penetration and the

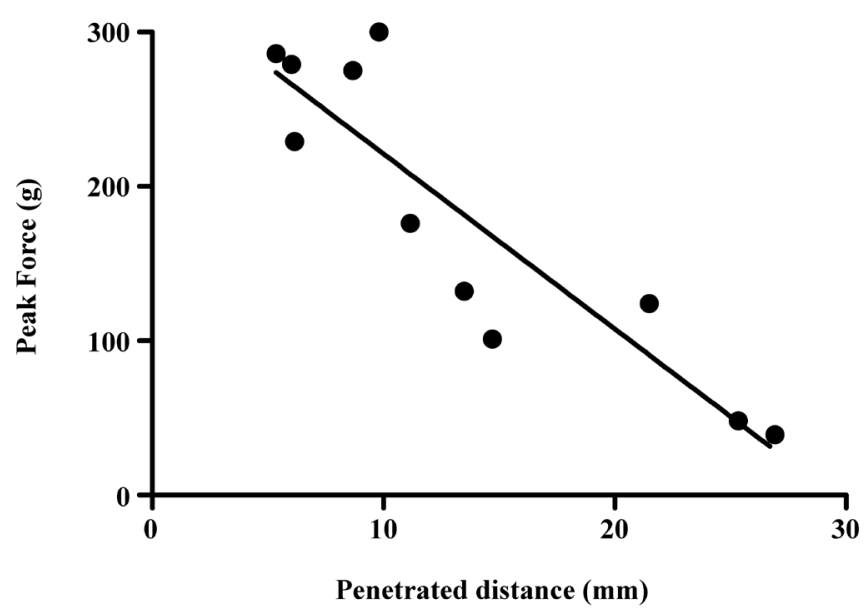

Fig. 3. Relationship between Penetration and Restitution
VAS score of palatability, which reached approximately 60 for $10 \mathrm{~mm}$ of the penetration and did not show a significant change.

\section{Discussion}

We sought to elucidate the acceptability of the appropriate physical properties of gummi drugs for clinical use by evaluating their relationship with the clinical sensory test parameters. First, we prepared 11 placebo gummi formulations with different gelatin and water contents, and then evaluated their physical properties. We confirmed that the physical properties of the gummi formulations could be controlled by adjusting the gelatin and water content. In addition, the good correlation between penetration and restitution were observed, and we used the penetration value as a measure of the physical properties in this study.

Next, we evaluated the elasticity and hardness of the gummi formulations in healthy volunteers using the VAS score. This is because elasticity and hardness are typical characteristics of gummi formulation that patients perceive when taking formulations, and they would impact their ability or willingness to take the formulations. The VAS score of elasticity was increased with increasing amounts of gelatin. On the other hand, although the VAS score of hardness increased with increasing gelatin content, it decreased with increasing water content (Fig. 4). We confirmed that adjusting the amount of gelatin and water is a useful strategy for controlling the elasticity and hardness of gummi formulations. The evaluation of the relationship between physical properties and the VAS scores of elasticity and hardness using clinical sensory tests, revealed good linear correlations (Figs. 5a, 5b). These results suggest that the penetration was well reflected by the hardness results of the clinical VAS scores, namely the physical characteristics that patients perceive when taking gummi formulations.

Interestingly, the correlation between penetration and the VAS score of overall palatability was not linear (Fig. 5c). The $\mathrm{L}$ and $\mathrm{M}$ formulations, which exhibited penetration values of not less than $8.7 \mathrm{~mm}$, showed VAS scores of overall palatability that reached approximately 60 , and the M3 formulation showed the highest score of 74.0. The evaluation of the prefer- (a)

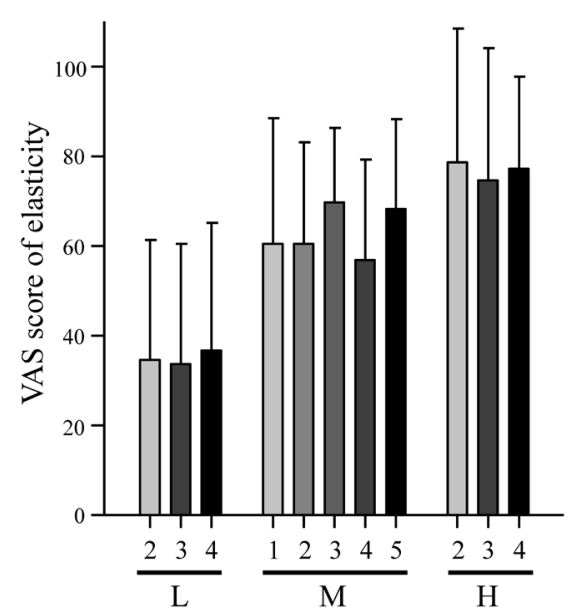

(b) (c)
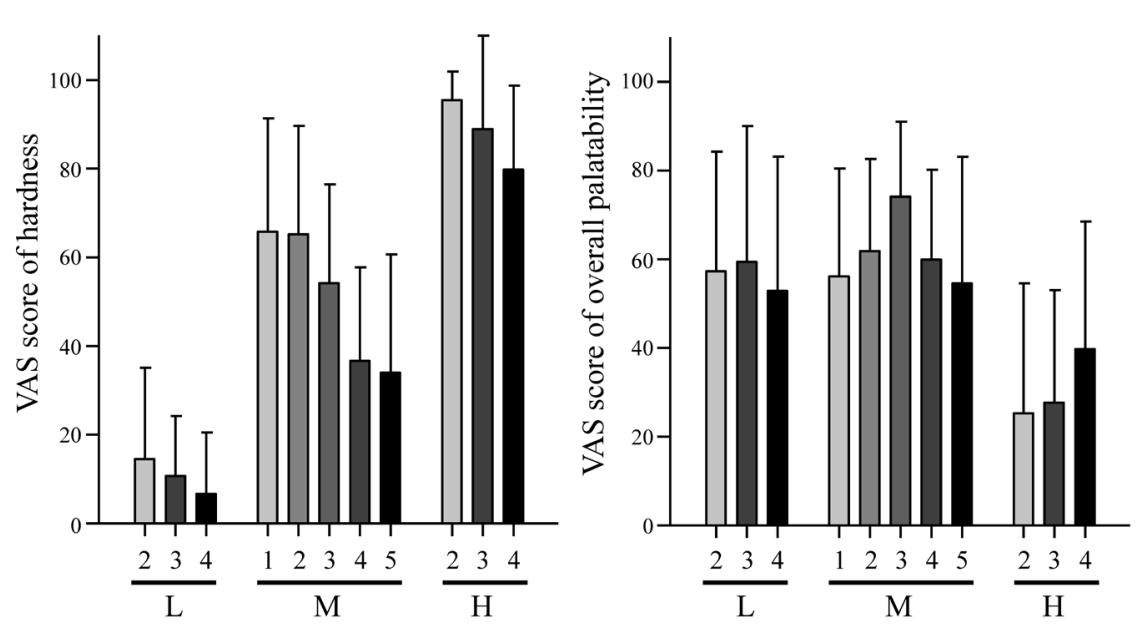

Fig. 4. Visual Analog Scale (VAS) Scores of (a) Elasticity, (b) Hardness, and (c) Overall Palatability for Each Gummi Formulation Columns are means \pm S.D. of three subjects. 
Table 3. Score of 5-Point Scale of Preference, Chewing Cycles, and Visual Analog Scale (VAS) Score of Ease of Swallowing in Clinical Sensory Test

\begin{tabular}{|c|c|c|c|c|}
\hline \multirow[b]{2}{*}{ Gummi formulation } & \multicolumn{2}{|c|}{ First trial } & \multicolumn{2}{|c|}{ Second trial } \\
\hline & $\begin{array}{c}\text { Score of 5-point scale of } \\
\text { preference }\end{array}$ & Number of preference (rate) & Chewing cycle & $\begin{array}{c}\text { VAS score of ease of } \\
\text { swallowing }\end{array}$ \\
\hline L2 & $3.5(1-5)$ & $8(50 \%)$ & $24.5 \pm 11.5$ & $73.8 \pm 25.8$ \\
\hline L3 & $3.5(1-5)$ & $8(50 \%)$ & $18.9 \pm 7.5$ & $89.0 \pm 12.2$ \\
\hline L4 & $3(1-5)$ & $6(38 \%)$ & $19.5 \pm 8.8$ & $87.0 \pm 12.7$ \\
\hline M1 & $3(1-5)$ & $7(44 \%)$ & $36.6 \pm 11.7$ & $64.0 \pm 19.0$ \\
\hline M2 & $4(1-5)$ & $10(63 \%)$ & $33.1 \pm 10.3$ & $71.0 \pm 16.6$ \\
\hline M3 & $4(2-5)$ & $13(81 \%)$ & $30.0 \pm 13.2$ & $73.7 \pm 13.7$ \\
\hline M4 & $4(2-5)$ & $10(63 \%)$ & $28.4 \pm 11.0$ & $75.0 \pm 14.0$ \\
\hline M5 & $3.5(1-5)$ & $8(50 \%)$ & $27.5 \pm 11.4$ & $78.4 \pm 12.1$ \\
\hline $\mathrm{H} 2$ & $1.5(1-4)$ & $2(13 \%)$ & $50.9 \pm 18.2$ & $47.8 \pm 28.5$ \\
\hline $\mathrm{H} 3$ & $2(1-4)$ & $2(13 \%)$ & $51.8 \pm 15.3$ & $39.3 \pm 25.3$ \\
\hline $\mathrm{H} 4$ & $2.5(1-4)$ & $4(25 \%)$ & $50.3 \pm 15.8$ & $39.4 \pm 22.0$ \\
\hline
\end{tabular}

Data of score of 5-point scale of preference are median (range), and data of chewing cycle and VAS score of ease of swallowing are means \pm S.D. of 16 subjects. Number of preference: number of subject who answered "prefer."

(a)

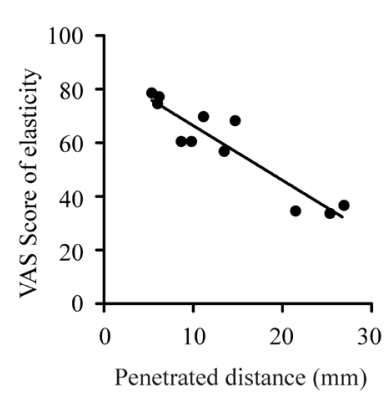

(b)

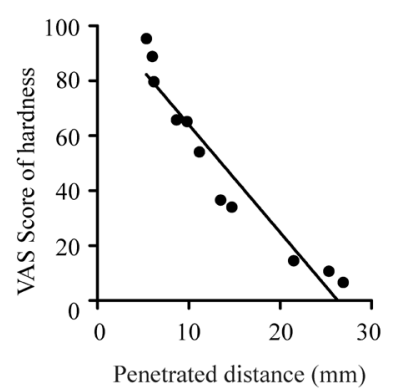

(c)

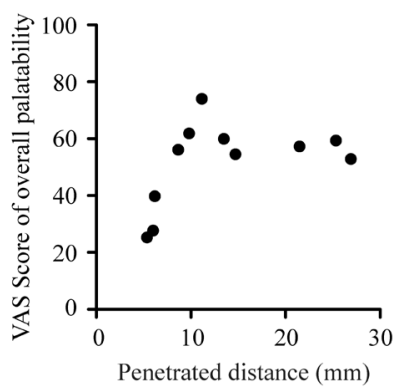

Fig. 5. Relationship between Penetration and (a) Elasticity, (b) Hardness, and (c) Overall Palatability

Values are means of three subjects.

ence of gummi formulations using a 5-point rating score revealed that $>50 \%$ of the volunteers "prefer" the M2, M3, and M4 formulations, which showed penetration values ranging from 9.8 to $13.5 \mathrm{~mm}$. The results of the VAS score of overall palatability and the 5-point rating score of preference indicate that the penetration of the preferred gummi formulation was in the above range and the most preferred gummi formulation was M3. Therefore, an appropriate level of hardness of gummi formulation is likely to exist, and suitable gummi formulations with clinically preferred physical characteristics could be prepared by adjusting the gelatin and water content and measuring their penetration.

Finally, in the second trial, we confirmed the number of the chewing cycles required before the gummi formulations could be swallowed comfortably, as well as the VAS scores of ease of swallowing (Table 3). The result of the clinical trial indicates that the required number of chewing cycles was approximately 20 and 50 for softer and harder gummi formulations, respectively. The disintegration time of the gummi formulations became shorter when the formulation samples were cut into small pieces, and those cut into not less than eight pieces showed an almost similar disintegration time (5-8 min, data not shown). Therefore, the various disintegration times of the formulations become almost comparable when the gummi formulations were consumed after they were chewed for some cycles. We expected the gummi formulations to show almost comparable VAS scores of ease of swallowing because the volunteers swallowed them after sufficient chewing to reduce the gummi particle size. ${ }^{14)}$ However, the VAS scores of the ease of swallowing differed among the gummi formulations and were lower for the $\mathrm{H}$ formulations than they were for the other formulations. We confirmed that the hardness of the gummi formulations affected the ease of swallowing and the harder gummi was difficult to swallow even after it was sufficiently chewed.

The human sensory tests were conducted using placebo gummi formulations and, therefore, a taste test was not conducted. The chewing cycles would affect the taste of the chewing gummi formulation to patients. If gummi formulations containing bitter tasting drugs were used in the human sensory tests, the window of the hardness of the gummi formulations would have been different from the present result of this test. It is very interesting that in determining the relationship between overall palatability and physical properties, taste is evaluated. However, in this human sensory test, we used gummi formulations of the same size and shapes. This is because it is also very important to confirm the effects of the shape and size of gummi formulation on overall palatability, as specified in the draft guidance for the FDA chewable development. $^{12)}$ 


\section{Conclusion}

We prepared 11 placebo gummi formulations with different hardness level and used human sensory tests to investigate them. We confirmed that the physical properties of gummi formulations could be controlled by adjusting the gelatin and water content, and penetration, which is one of the critical physical properties, is well reflected by the hardness as revealed by the clinical VAS scores. Furthermore, these are the physical characteristics that patients experience when taking gummi formulations. However, the relationship between penetration and the VAS score of overall palatability showed no linear correlation, and an appropriate window of the hardness of gummi formulation is likely to exist. An appropriate gummi formulation with clinically preferred physical characteristics could be prepared by adjusting the gelatin and water content and measuring its penetration. Additionally, we confirmed that the chewing cycles of the gummi formulations were from 20 to 50 . Chewed gummi formulations would show almost similar disintegration times because samples of the 11 gummi formulations cut into not less than eight pieces showed an almost comparable disintegration time.

Acknowledgments The authors are grateful to Ms. Wakana Shibakiri, Mr. Shuta Seki, and Mr. Miohiko Sugimoto for excellent technical assistance.

Conflict of Interest FN are employee of Kissei Pharmaceutical Co., Ltd. (Tokyo, Japan). SU and NN received a research Grant from Kissei Pharmaceutical Co., Ltd., Takeda Consumer Healthcare Co., Ltd. (Tokyo, Japan), and Otsuka Pharmaceutical Co., Ltd. (Tokyo, Japan). NN serves as a consultant to Kissei Pharmaceutical Co., Ltd., Otsuka Pharmaceutical Co., Ltd., and Shiseido Japan Co., Ltd. (Tokyo, Japan).

\section{References and Notes}

1) Ocak E., Kocaoz D., Acar B., Int. J. Pediatr. Otorhinolaryngol., 100, 194-197 (2017).

2) van Rein N., de Geus K. S., Cannegieter S. C., Reitsma P. H., van der Meer F. J. M., Lijfering W. M., Pharmacoepidemiol. Drug Saf. (2017). doi: 10.1002/pds.4346

3) Bosworth H. B., Brown J. N., Danus S., Sanders L. L., McCant F., Zullig L. L., Olsen M. K., Am. J. Manag. Care, 23, 280-286 (2017).

4) Ikematsu Y., Uchida S., Namiki N., Chem. Pharm. Bull., 63, 156163 (2015).

5) Sheshala R., Khan N., Chitneni M., Darwis Y., Arch. Pharm. Res., 34, 1945-1956 (2011).

6) Hanawa T., Watanabe A., Tsuchiya T., Ikoma R., Hidaka M., Sugihara M., Chem. Pharm. Bull., 43, 284-288 (1995).

7) Suzuki H., Onishi H., Hisamatsu S., Masuda K., Takahashi Y., Iwata M., Machida Y., Int. J. Pharm., 278, 51-61 (2004).

8) Prajapati S. T., Mehta A. P., Modhia I. P., Patel C. N., Int. J. Pharm. Investig., 2, 176-182 (2012).

9) Namiki N., Takagi N., Yuasa H., Kanaya Y., Biol. Pharm. Bull., 21, 87-89 (1998).

10) Namiki N., Negishi T., Katou J., Kaneko M., Ishikura T., Nagata F., Jpn. J. Pharm. Health Care Sci., 29, 92-99 (2003).

11) Uchida S., Hiraoka S., Namiki N., Chem. Pharm. Bull., 63, 354-360 (2015).

12) Food and Drug Administration, Center for Drug Evaluation and Research (CDER), "Guidance for Industry: Quality attribute Considerations for Chewable Tablets," U.S. Department of Health and Human Service, USA: 〈htps://www.fda.gov/downloads/Drugs/ Guidances/UCM507098.pdf), cited 12 December, 2016.

13) Horio T., Kawamura Y., J. Oral Rehabil., 16, 177-183 (1989).

14) Engelen L., Fontijn-Tekamp A., van der Bilt A., Arch. Oral Biol., 50, 739-746 (2005). 